\title{
Relationship between the Corynebacterium pseudotuberculosis, phospholipase D inoculation and the fertility characteristics of crossbred Boer bucks
}

\begin{abstract}
The aim of this study was to investigate the effects of Corynebacterium pseudotuberculosis and phospholipase D (PLD) on buck's fertility, specifically, testosterone concentration, scrotal circumference and semen quality. Crossbred Boer goats $(n=13)$ aged 12-14 months were divided into three groups. The first group $(n=3)$ was inoculated with one ml sterile phosphate buffer saline subcutaneously into subaxillary area as the control. The second group (n=5) was inoculated with live C. pseudotuberculosis $1 \times 10^{9}$ cfu subcutaneously into subaxillary area. The third group $(\mathrm{n}=5)$ was inoculated with PLD $1 \mathrm{ml} / 20 \mathrm{~kg}$ BW intravenously into jugular vein. Blood collection was done twice a week over a period of three months for testosterone analyses. Semen was collected using electro-ejaculator once every two weeks whilst scrotal circumference was measured once every three weeks. Semen volume, semen $\mathrm{pH}$ and sperm progressive motility, concentration, live/dead percentage and morphology were evaluated. Bucks were euthanized three months' post inoculation and histopathological examination of the testicles and epididymis was performed. The results showed seven folds' significant decrease $(P<0.05)$ in testosterone concentration in both $C$. pseudotuberculosis and PLD inoculated groups compared to the control. Semen volume, percentage of dead/live and abnormal sperm morphology showed significant increase $(\mathrm{P}<0.05)$ in both inoculated groups compared to the control. The following sperm defects were observed; tapered sperm, decapitated and knobbed head, thickened midpiece, distal and proximal droplet, sterilizing tail and folded tail. Scrotal circumference, semen $\mathrm{pH}$, semen wave pattern, sperm motility and concentration showed significant decrease $(\mathrm{P}<0.05)$ in both inoculated groups compared to the control. The testicles showed varied degrees of degeneration and necrosis with shrunken seminferous tubules. In conclusion, testosterone concentration, scrotal circumference and semen quality were negatively affected in both inoculated groups. Moreover, percentages of live/dead and abnormal sperm morphology were also increased. The results suggest that both $C$. pseudotuberculosis and PLD had detrimental effects on buck's fertility.
\end{abstract}

Keyword: Boer; Buck; Goat; Corynebacterium pseudotuberculosis; Phospholipase D; Fertility 